\title{
AUTOMATIC RELATIONSHIP-HARM ASSOCIATIONS AND INTERPERSONAL TRAUMA INVOLVING CLOSE OTHERS
}

\author{
Anne P. DePrince \\ University of Denver \\ Michelle Shanahan \\ University of Illinois Medical Center
}

Melody D. Combs

University of Colorado Denver

\begin{abstract}
Women exposed to violence early in life are at risk of revictimization. The interpersonal schema hypothesis of revictimization proposes that revictimized women will be more likely to hold negative expectations about intimate relationships, including expectations that relationships involve harm, relative to singly or nonvictimized women. To test the interpersonal schema hypothesis, we used the implicit lexical decision task to examine automatic associations between relationship and harm concepts among college women who reported histories of no, single, or multiple types of interpersonal trauma involving close others (e.g., family member, partner). Women exposed to multiple types of interpersonal trauma involving close others showed stronger relationship-harm associations than singly or nonexposed women. Relationship-harm associations predicted the number of interpersonal trauma types (e.g., sexual, physical trauma) involving close others reported by participants, but were unrelated to posttraumatic stress disorder (PTSD) symptoms. Findings suggest that relational schemata held by women exposed to multiple types of interpersonal trauma involving close others include concepts of harm, which may have implications for how multiply victimized women behave in and think about intimate relationships.
\end{abstract}

Women exposed to interpersonal trauma such as sexual assault in their youth are at increased risk of exposure to interpersonal trauma later in life (e.g., Arata, 2002; Classen, Palesh, \& Aggarwal, 2005; Cloitre, Tardiff, Marzuk, Leon, \& Potera, 1996; Gidycz, Hanson, \& Layman, 1995), particularly when early interpersonal trauma is perpetrated by a close other such as a parent or caregiver (e.g., Classen et al., 2005; DePrince, 2005; Noll, Horowitz, Bonanno, Trickett, \& Putnam, 2003). Recognizing the serious public health problem posed by such revictimization, several researchers have called for increased research into potential

Anne P. DePrince, Department of Psychology, University of Denver; Melody D. Combs, Department of Psychology, University of Colorado Denver; Michelle Shanahan, University of Illinois Medical Center.

During the compilation of this article Melody D. Combs and, Michelle Shanahan were affiliated with the Department of Psychology at the University of Denver.

Thank you to Eileen Zurbriggen for providing feedback on the priming task design and stimuli, Jennifer J. Freyd for comments on an earlier version of the manuscript, and undergraduate research assistants for project assistance.

Address correspondence and reprint requests to: Anne P. DePrince, Department of Psychology, 2155 S. Race Street, Denver, CO 80208. E-mail: adeprinc@du.edu mechanisms that may increase risk (Classen et al., 2005; Cloitre, Cohen, \& Scarvalone, 2002).

The interpersonal schema hypothesis of revictimization proposes that revictimized women hold negative expectations of others in relationships, including expectations of harm, relative to singly or nonvictimized women (Cloitre, 1998; Cloitre et al., 2002). A schema is an automatically activated set of associations that can have an impact on thoughts and behavior (see Cloitre et al., 2002; Lindgren, Shoda, \& George, 2007). Several theorists have proposed that interpersonal traumas involving close others early in life can disrupt the development of healthy schemas and attachment (Cloitre et al., 2002; Freyd, DePrince, \& Gleaves, 2007). For example, a child who tries to elicit attachment from caregivers and is met with abusive behavior may develop templates for future relationships that link relationships and harm. In particular, Cloitre et al. (2002) argued that children exposed to abuse by caregivers and close others may develop schemata that "reflect the learned contingency that to be interpersonally engaged means to be abused, and that abuse is a way to be connected" (p. 92). The interpersonal schema hypothesis of revictimization suggests that women whose relationship schemata include concepts of harm (i.e., harm is expected to be part of relationships) may be at increased risk of exposure to additional interpersonal traumas. Alternatively, women who repeatedly experience interpersonal traumas involving close others may 
develop stronger associations between relationships and harm as a consequence of learning, relative to their singly or nonexposed peers. Thus, relationship-harm schemata could serve as either risk factors for and/or consequences of revictimization risk.

Support for the interpersonal schema hypothesis largely comes from studies using explicit, self-report methods (Cloitre et al., 2002; Jurgens, 2005). For example, women who reported sexual violence before and after age 18 were more likely than singly victimized women to generalize negative relationship schemata from childhood to adulthood and to hold negative expectations about adult relationships. Self-report methods tap women's explicit beliefs about relationships; however, participants often have difficulty accurately reporting on their own information processing (see Zurbriggen, 2000). Implicit tasks that do not require selfreport may be particularly valuable when studying valueladen topics, such as violence and harm. Further, schemata involve automatic associations that can occur outside of conscious awareness; therefore, tests of schema theory should include implicit methods that can tap automatic processes outside conscious awareness. Researchers have used implicit methods to examine automatic links between concepts such as power and sex, finding that these associations exist among men likely to engage in abusive or aggressive behavior (e.g., Bargh, Raymond, Pryor, \& Strack, 1995; Bushman, 1998; Kamphius, deRuiter, Janssen, \& Spiering, 2005; Mussweiler \& Forster, 2000; Zurbriggen, 2000). These studies have largely relied on a priming paradigm using the lexical decision task (LDT) to test the strength of semantic associations (e.g., power-sex, sex-aggression) relevant to understanding the consequences of or risk factors for aggressive behavior.

The priming LDT examines the time required for participants to respond to a target stimulus following a prime. Participants' reaction times are faster when the target stimulus is part of a network of associative links (or schema) activated by the prime stimulus compared to when the target is semantically unrelated to the prime. Participants are asked to decide whether two words presented together (one above the other) are both real words (e.g., "cat" is a real word but "pritem" is not). Because the word "cat" appears first, it is the prime. If the prime "cat" activates an associative network having to do with small, furry animals, participants respond faster when the word "dog" appears as the target than the word "nail." The decrease in reaction time when the target is "dog," relative to other unrelated targets, offers an implicit measure of the schema or interconnected associations activated by "cat."

To study the interpersonal schema hypothesis of revictimization, we tested whether the associative network primed by relationship words includes harm concepts; this is referred to as relationship-harm priming. If relationshipharm priming occurs, participants will be faster when "lover" (a relationship prime) is followed by "assault" (a harm target), versus "tomato" (an unrelated, neutral target).
We can also test whether harm primes activate relationship concepts (referred to as harm-relationship priming). If so, participants will be faster when "exploit" (a harm prime) is followed by "husband" (a relationship target) versus "fruit" (an unrelated, neutral target).

In one of the earliest applications of the priming LDT to aggression, Bargh et al. (1995) found that men who scored high on a measure of sexual aggressiveness showed powersex priming, but not sex-power priming. That is, the presentation of power words led to automatic associations with sex words. However, the reverse was not true: Sex words did not lead to automatic associations with power. Thus, this study pointed to a unique pattern of unidirectional powersex associations among men high in self-reported sexual aggression, relative to others. Bargh et al. argue that automatic power-sex association can be either a consequence or risk factor for engaging in sexual harassment or aggression. As a consequence of harassment, the power-sex link may develop because associations between power and sex are learned and rehearsed when one engages in sexual harassment. Alternately, the preexistence of power-sex links may increase the likelihood of engaging in sex-related behaviors when concepts of power are activated. Several studies have replicated and extended Bargh's initial unidirectional findings in relation to aggression (e.g., Mussweiler \& Forster, 2000; Zurbriggen, 2000).

The interpersonal schema hypothesis implies that automatic associations between relationship and harm could develop as either a consequence of repeated victimization and/or a risk factor for additional victimizations. As a consequence of exposure to multiple interpersonal traumas perpetrated by a close other, relationship-harm priming could reflect learning that occurs following repeated exposure to harm in relationships. From this perspective, women learn that interpersonal harm is part of the schema, or set of automatic expectations, for relationships. These same relationship-harm associations could become a risk factor for future interpersonal traumas to the extent that concepts of relationships include expectations of harm. Thus, women whose schemas for relationships include harm might be more likely to expect harm in relationships. Therefore, we predicted that women exposed to multiple interpersonal traumas by close others would show faster response times when relationship primes preceded harm targets, relative to other conditions in which relationship primes and harm targets were presented separately (e.g., husband-tomato; bird-assault). According to the interpersonal schema hypothesis of revictimization, the singly and nonvictimized women were not expected to show this priming pattern, indicating that these groups do not hold views of relationships as including harm.

Based on previous unidirectional priming findings in the aggression literature, we predict that harm concepts would not automatically activate relationship concepts (harm-relationship priming). When Mussweiler and Forster (2000) failed to find aggression-sex priming, they 
reasoned that many aggressive situations do not include sex content; thus, broad aggression concepts should not automatically activate specific sex concepts. Harm, like aggression, is a broad concept; therefore, we reasoned that this broad concept would not be automatically linked with specific relationship concepts. Alternately, automatic links between harm and relationships may be developed through media images or gender socialization, rather than being specific to the experience of multiple interpersonal traumas. Thus, to the extent that harm is caused by another person and, therefore, activates associative links to relationship concepts, we expected harm-relationship priming to be similar across the no, single, and multiple close victimization groups.

We approached data analysis in two ways. First, we tested whether women showed relationship-harm priming in relation to their self-reported interpersonal trauma exposure (no, single-, multiple-interpersonal trauma involving close others). This first test was designed to evaluate simply whether priming occurred. Priming was defined as a decrease in reaction time when relationship primes and harm targets appeared together, relative to when the relationship primes and harm targets appeared separately (e.g., relationship-neutral or neutral-harm pairs). Using equations that will be explained in more detail below, a priming score of zero indicated that participants performed no differently when relationship primes and harm targets were presented together, relative to other conditions in which the words appeared separately. Thus, scores greater than zero indicated that priming had occurred.

Second, we asked whether priming scores could explain variance in the number of close victimizations women reported, even after controlling for self-reported symptoms. Though victimization group was the independent variable in the first set of analyses, we used the number of victimizations as an outcome in this set of analyses to evaluate the potential relevance of priming to understanding revictimization by close others. Revictimization was defined as the number of types of interpersonal traumas (e.g., sexual assault, physical assault, witnessing harm) involving close others reported by participants. Based on Cloitre's interpersonal schema hypothesis, we predicted that the automatic relationship-harm priming would explain unique variance in the total number of interpersonal trauma types involving close others reported; we predicted that harm-relationship priming would not contribute to the model. To demonstrate that relationship-harm priming explains unique variance in the number of interpersonal trauma types involving close others, it was important to control for symptoms that could also affect information processing because trauma-related symptoms have been associated with information processing alterations (e.g., DePrince \& Freyd, 2004; McKenna \& Sharma, 1995; Williams, Mathews, \& MacLeod, 1996). Therefore, we included PTSD symptom severity, dissociative experiences, and general distress in our regression model.

\section{METHOD}

\section{Participants}

Ninety-nine undergraduate females were recruited into a "Stress and Attention" study. Due to computer problems, we missed priming data for two participants. Thus, we present data from 97 participants (age $M=19.93, S D=$ 1.64) who reported belonging to the following ethnic and racial groups: $77 \%$ Caucasian, 6\% Asian American, 2\% African American, 2\% Native American, 2\% another group not listed, and 8\% Latino/Hispanic. Participants received extra credit in their undergraduate psychology courses as compensation for their time.

\section{Materials: Self-Report Measures}

The Brief Betrayal Trauma Survey (BBTS; Goldberg \& Freyd, 2006) is a 12-item self-report measure that assesses exposure to interpersonal (e.g., sexual assault) and noninterpersonal (e.g., natural disaster) traumas using behaviorally defined items. Participants were asked if they experienced each event before age 18 or at/after age 18; thus participants could report up to 24 potentially traumatic events. Nine items that assessed interpersonal traumas involving close others were used to characterize women's experiences of interpersonal trauma. Sample items of interpersonal trauma involving close others include: "Witnessed someone with whom you were very close deliberately attack another family member so severely as to result in marks, bruises, blood, broken bones, or broken teeth" and "You were deliberately attacked that severely [so severely as to result in marks, bruises, blood, broken bones, or broken teeth] by someone with whom you were very close."

The BBTS was scored as follows. First, the total number of interpersonal trauma types involving close others reported before age 18 (including witnessing harm among close others and physical, sexual, and/or emotional abuse perpetrated by close others) and age 18 or older (physical, sexual, and/or emotional abuse perpetrated by close others) were calculated. Based on the tally of interpersonal trauma types involving close others, participants were further categorized into one of three exposure groups: no, single, or multiple interpersonal trauma exposure. Analyses were conducted using both the continuous (tally of interpersonal trauma types involving close others) and categorical variables (no, single, or multiple interpersonal traumas). Women who denied interpersonal traumas, but who reported exposure to noninterpersonal events (such as a natural disaster or serious motor vehicle accident) were included in analyses involving PTSD symptoms because they could have developed such symptoms related to the noninterpersonal event exposure.

The Trauma Symptom Checklist-40 (TSC-40; Briere \& Runtz, 1989) assesses symptoms commonly associated with the experience of traumatic events across six domains: 
depression, dissociation, anxiety, sexual problems, sleep disturbance, and sexual trauma symptoms (e.g., bad thoughts during sex, fear of men). Participants were asked to indicate how frequently they experienced each of 40 items on a scale of 0 (never) to 3 (often). The TSC-40 is scored by summing responses and has been shown to have good reliability and validity (e.g., Elliott \& Briere, 1992). Sample items include "anxiety attacks" and "trouble getting along with others." Norm data for determining clinical significance of symptoms are not available.

The Dissociative Experiences Scale (DES; Bernstein \& Putnam, 1986) is a 28-item self-report measure that assesses dissociative experiences. Participants indicate what percentage of time they experience each of the 28 items, such as "Some people find that they become so involved in a fantasy or daydream that it feels as though it were really happening to them." The DES has been shown to have good validity and reliability. The measure is scored by taking an average across the 28 items for each participant. In a nonclinical sample, scores greater than or equal to 20 are considered high and potentially indicative of dissociationrelated problems (Bernstein \& Putnam, 1986).

The Revised Civilian Mississippi Scale for PTSD (RCMS; Norris \& Perilla, 1996) is a self-report measure of PTSD symptom severity derived from the original version of the scale used in veteran populations (Norris \& Perilla, 1996). The RCMS contains 30 items and has been shown to be a reliable and valid measure of PTSD symptom severity across a variety of traumas (Norris \& Perilla, 1996). Participants rate items on a scale of 1 (not at all true) to 5 (extremely true). Sample items include "Since the event, unexpected things make me jump" and "I try to stay away from anything that will remind me of things which happened during the event." The scale developers report that mean scores for trauma-exposed community participants on the RCMS ranged from 1.63 to 1.97 across two samples. Among participants who reported both life-threat and injury during a trauma, mean scores on the RCMS were 2.2, and $37 \%$ of the sample met full symptom criteria for PTSD (Norris \& Perilla, 1996).

\section{Materials: Priming Stimuli}

Three types of words were used in the priming task: neutral, harm, and relationship. Length of word was matched across lists. ${ }^{1}$ Replicating Zurbriggen (2000), neutral words were selected from Meyer, Schvaneveldt, and Ruddy's (1975) list of semantically related pairs. Nonwords were replicated from Zurbriggen (2000), and words were matched across categories for length. Relationship and harm words were generated for the current study, based in part on words used in previous studies (e.g., DePrince \& Freyd, 2004; McNally, Metzger, Lasko, Clancy, \& Pitman, 1998). Additional harm words and relationship words were generated from Webbased searches. Because of concerns that harm words (e.g., agony, betrayed) could just as likely represent aspects of relationships, we asked six research assistants not involved in this study to rate all words on the degree to which they represented (1) violence and (2) relationships on a scale of 0 (not at all) to 4 (entirely). Paired $t$-tests revealed the harm words were rated as more representative of violence $(M=$ $2.46, S D=.46)$ than relationships $(M=.05, S D=.09)$, $t(5)=14.06, p<.001$, and the relationship words were rated as more representative of relationships $(M=3.31$, $S D=.63)$ than violence $(M=.77, S D=.61), t(5)=7.47$, $p=.001$. Effect sizes were large (Hedges $g=6.36$ and 3.54 , respectively).

\section{Design}

The process for assigning words to trial types and blocks was replicated from Zurbriggen (2000). All trial types were made up by pairs of words. Three nonword conditions (in which one or both words were nonwords) were viewed by participants, but not used in analyses. Ten word trial types (in which both words in the pair were English words) were used to calculate priming scores, including: Harm-Relationships (HR)a, Harm-Relationships (HR)b, Neutral-Relationship (NR), Harm-Neutral (HN), Semantically Unrelated (UR) 1 , Relationship-Harm (RH)a, Relationship-Harm (RH)b, Neutral-Harm (NH), Relationship-Neutral (RN), and Semantically Unrelated $(\mathrm{UR})_{2}$. Several trial types are logically equivalent (e.g., HRa and HRb), but were set up separately in order to allow all words to appear exactly three times (see below). Semantically unrelated pairs always comprised neutral stimuli (e.g., lettuce-answer).

Three experimental blocks contained 48 (24 word and 24 nonword) trials, each made up of unique words. Trial types included the following mutually exclusive primetarget combinations: HR, NR, HN, RH, NH, RN, and UR. Each word appeared only once in a single experimental block. Thus, words were randomly assigned to be either primes or targets in three trial types, only one of which was used in any experimental block. For example, the word "slap" was assigned to be the prime in $\mathrm{HRa}, \mathrm{HRb}$, and $\mathrm{HN}$ trials. If "slap" appeared in a HRa trial in experimental block 1, then HRb and HN trials in that same block did not use the word "slap," but instead used another harm word. Each experimental block was repeated three times, with each word appearing in a new trial type with a new pair. Thus, the word "slap" might appear in experimental block la as the HRa trial, in experimental block $1 \mathrm{~b}$ as the $\mathrm{HRb}$ trial, and in experimental block $1 \mathrm{c}$ as the $\mathrm{HN}$ trial; in each experimental block, the corresponding pair was different (e.g., cherish, beloved, food). Experimental blocks were not repeated until the other two experimental blocks had been administered, thus maximizing time between re-presentation of any stimulus. Three versions of the experiment using different experimental block orders were used. Experimental block order was not significantly related to priming outcomes ( $p>.15$ for all). The order of 
individual trials within each block was randomized for each participant.

The following equations were used to calculate priming (see Zurbriggen, 2000, for detailed mathematic justification of these calculations). These equations remove the effects of presenting harm and relationship words in other combinations (e.g., harm words that appeared in $\mathrm{HN}$ trials). This is important because reaction time could be affected by simply seeing a harm word appear on the screen, regardless of whether that word is primed by a relationship word. For example, women might slow down when the word "rape" appears because it is surprising or distracting to see on the computer. Therefore, we used these calculations to isolate the decrease in reaction time caused by pairing relationship and harm words relative to other conditions where those words appeared, therefore controlling for any general effects of seeing these particular words in the course of the experiment. Thus, we were able to examine the specific speed-up in processing caused by the appearance of relationship and harm words together, relative to other combinations of words. Equation 1: harm-relationship priming $=\mathrm{NR}-[(\mathrm{HRa}+\mathrm{HRb}) / 2]-$ $\mathrm{UR}_{1}+\mathrm{HN}$. Equation 2: relationship-harm priming = $\mathrm{NH}-[(\mathrm{RHa}+\mathrm{RHb}) / 2]-\mathrm{UR}_{2}+\mathrm{RN}$. A harm-relationship priming score of zero indicates that the reaction time when a relationship word followed a harm prime did not differ relative to other conditions where harm words were presented as the primes. That is, a score of zero indicates there is no decrease in reaction time caused by the words presented together relative to reaction time when the harm and relationship words are presented without one another. Higher scores represent facilitation in response to the target caused by the prime. For example, higher relationshipharm priming scores indicate facilitation (or decrease in reaction time) when harm words are presented after (or primed by) relationship words, after taking into account reaction time in other conditions where harm and relationship words appear.

\section{Procedure}

Prior to data collection, all procedures were approved by the institutional review board where this research took place. Participants were tested individually by an undergraduate or graduate research assistant in a quiet, private room. The experimenter explained the study procedure to the participants by stating that they would be asked to complete a series of tasks on the computer consisting of an experimental task and some questionnaires. Participants were told that some of the questionnaires asked about stressful life events, such as physical trauma, and that they could skip any questions they did not want to answer throughout the entire experiment. Participants were also assured that their responses would be recorded only by a randomly generated number that would not be stored with their name. Following the consent procedures, the participants heard instructions for the computer-based priming task. Participants were told that, for each block, they would be presented with several trials in which two words would appear on the computer screen at the same time, one on top of the other. For each pair of words presented, they were to decide if the words were real words (e.g., house) or nonwords (e.g., dorb): They were to press a button on the keyboard marked "W" if both words were real words and a button marked "NW" if either of the words were nonwords. Participants were directed to respond as quickly and as accurately as possible.

The LDT was presented using EPrime software (version 1.1). Participants saw two practice blocks prior to the nine experimental blocks. Practice blocks included three harm and relationship words. For each trial, a fixation cross appeared in the center of the screen for $400 \mathrm{~ms}$. Following a 150-ms inter-trial interval, two words appeared in the center of the screen, with one word appearing slightly above where the fixation had been and one below. Stimuli remained on the screen until participants made a response, for a maximum of $5,000 \mathrm{~ms}$, followed by a 1,000-ms inter-trial interval. At the end of each block, participants saw the percent correct and average response time for the block.

After the experimental task, the experimenter explained to the participants how to navigate through the computer questionnaire screens, which were programmed with $\mathrm{Mi}$ crosoft Access. The experimenter told the participants that their responses would be stored only by their participant number and that the file would automatically close when they were finished making their responses and clicked the "Done" button. The experimenter sat on the opposite side of the room with his/her back turned to the participants throughout the duration of the experimental task and questionnaire to ensure privacy while remaining available for questions. Finally, the experimenter debriefed the participants and welcomed questions.

\section{RESULTS}

\section{Self-Report Data}

BBTS data was missing for one participant. Of the remaining 96 participants, all but 12 women reported exposure to at least one event. Turning to the nine items used to identify interpersonal traumas involving close others, 42 women reported no, 13 reported one, and 41 reported multiple interpersonal traumas involving close others. Descriptive statistics for the self-report symptom measures by exposure group are reported in Table 1. Women who reported exposure to some form of trauma other than interpersonal trauma involving close others are also included in the PTSD symptom severity analyses.

Women reported an average of $1.65(S D=1.97)$ interpersonal traumas involving close others (range 0-8). Not surprisingly, the distribution was skewed because 
Table 1

Mean $(S D)$ by Group for Symptom Measures

\begin{tabular}{lccc}
\hline & \multicolumn{3}{c}{ Close interpersonal trauma } \\
\cline { 2 - 4 } & No & Single & Multiple \\
\hline Dissociative & $10.10(7.48)$ & $11.05(5.51)$ & $11.19(7.54)$ \\
experiences & $n=42$ & $n=13$ & $n=40$ \\
General distress & $20.07(11.69)$ & $29.38(13.22)$ & $30.12(14.81)$ \\
& $n=42$ & $n=13$ & $n=41$ \\
PTSD symptom & $1.70(.54)$ & $1.84(.58)$ & $1.94(.49)$ \\
severity & $n=28$ & $n=13$ & $n=38$ \\
\hline
\end{tabular}

Note. Dissociative experiences were assessed by the Dissociation Experiences Scale, general distress was assessed by the Trauma Symptom Checklist, and posttraumatic stress disorder (PTSD) symptom severity was assessed by the Revised Civilian Mississippi Scale for PTSD. PTSD symptom scores in the "no close interpersonal trauma group" are reported only for those women who reported another type of trauma exposure. Because of missing data due to participant failure to complete questionnaires or computer problems, $n$ for each cell is also reported.

42 women reported no such exposure. Thus, a natural log transformation was performed on this variable for the regression analyses that used the continuous number of traumas reported. These analyses were run with both the untransformed and transformed data; because findings were comparable, only the untransformed data are reported here.

\section{Reaction-Time Data}

Data were cleaned in several steps, similar to procedures used in previous studies (i.e., Holland, Hendriks, \& Aarts, 2005; Vitevitch, 2007). First, all trials for which participants made errors were deleted. Second, reaction times of less than $200 \mathrm{~ms}$ or greater than 2,000 ms were deleted because of concerns that these were likely to be invalid responses (e.g., anticipatory response before stimuli were actually read or distraction leading to failure to respond in a timely fashion, respectively). Next, data were examined for outliers at the individual participant level prior to calculating means for each condition. Reaction times that exceeded 2.5 standard deviations above the mean for each participant in each condition were deleted; by this criterion, an average of $1.95 \%$ ( $S D=.78$ ) of trials were deleted. Finally, group means for each condition by victimization group were calculated (see Table 2). Average (standard error) priming scores by victimization group are reported in Figure 1.

We first tested whether each of the three groups showed priming, defined as average priming scores that differed from zero. This is an important first step because later group comparisons could be significant even though individual groups might not have shown priming, defined as facilitation that differs from zero. The multiple interpersonal trauma group showed relationship-harm priming, $t(40)=$ 2.13, $p=.04$; this group's harm-relationship priming did not reach conventional significance levels, but a trend was observed, $t(40)=1.97, p=.06$. Neither the no nor the single close interpersonal trauma groups showed evidence of priming in either condition; the difference between their respective relationship-harm and harm-relationship priming scores and zero was not significant.

We next tested our between-group hypotheses using planned contrast weights, which were assigned based on hypothesized means (Loftus, 1996). Using contrast weights of 2 (multiple close interpersonal traumas), -1 (single close interpersonal trauma), and -1 (no close interpersonal trauma) to compare the latter two groups to the multiple close interpersonal trauma group, the contrast was significant, $t(93)=2.26, p=.03$, for relationship-harm priming. This same contrast was not significant for harmrelationship priming, $t(93)=1.28, p=.20$.

We next examined whether priming scores explained unique variance in the number of close interpersonal traumas reported, while controlling for symptoms of general distress as measured by the TSC, dissociative experiences as measured by the DES, and PTSD symptom severity as measured by the RCMS. Zero-order correlations between number of close events reported, symptom measures, and priming are reported in Table 3. To examine the full sample, the first model did not include PTSD symptom scores because those could be calculated only for participants who reported some form of trauma exposure. Thus, general

Table 2

Means $(S D)$ by Trial Type and Group

\begin{tabular}{lccc}
\hline Trial type (prime-target) & No close & Single close & Multiple close \\
\hline Neutral-Relationship (NR) & $756.66(142.16)$ & $726.25(125.23)$ & $783.70(122.55)$ \\
Neutral-Harm (NH) & $807.44(171.85)$ & $783.62(158.18)$ & $848.40(137.18)$ \\
Relationship-Neutral (RN) & $798.71(187.86)$ & $746.24(152.60)$ & $830.97(118.68)$ \\
Relationship-Harm (RH) & $848.72(194.00)$ & $791.61(162.44)$ & $879.19(160.21)$ \\
Harm-Neutral (HN) & $836.22(191.13)$ & $771.64(167.24)$ & $858.48(131.98)$ \\
Harm-Relationships (HR) & $833.10(181.68)$ & $782.59(164.56)$ & $870.16(143.04)$ \\
Semantically Unrelated (UR) & $735.30(129.87)$ & $730.44(106.30)$ & $724.51(133.23)$ \\
Semantically Unrelated (UR) & $779.74(144.42)$ & $777.24(137.65)$ & $751.02(124.39)$ \\
\hline
\end{tabular}

Note . No close interpersonal trauma $=$ no close, single close interpersonal trauma $=$ single close, and multiple close interpersonal traumas $=$ multiple close. 


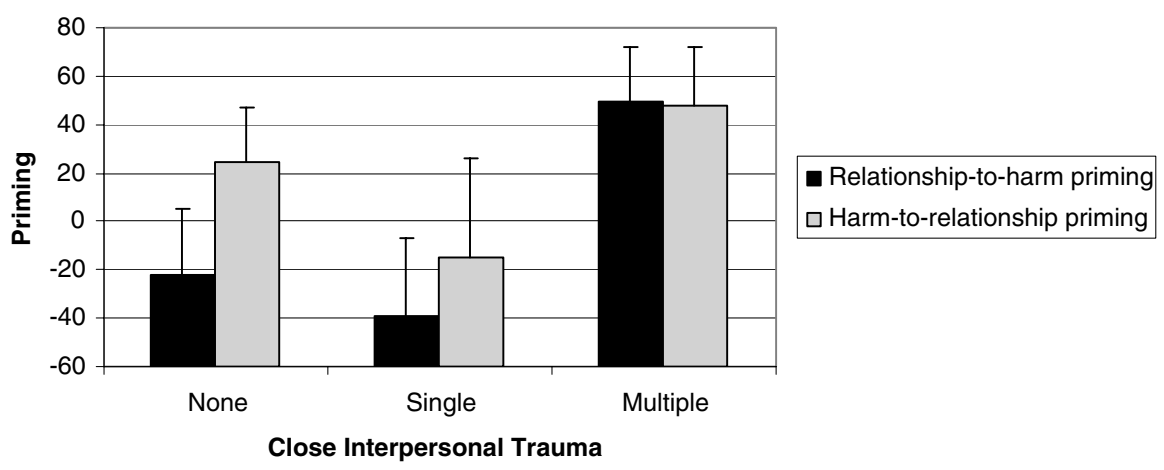

Note. Higher scores indicate a speed-up or facilitation of reaction time when the primes and targets appear together versus in other combinations. Specifically, higher scores in the relationship-harm priming condition indicate facilitation when relationship primes are followed by harm targets relative to when relationship primes and harm targets appear with other words (e.g., husband-tomato; bird-assault). Likewise, higher scores in the harm-relationship priming condition indicated facilitation when harm primes are followed by relationship targets relative to other conditions.

Fig. 1. Relationship-harm priming and harm-relationship priming (with standard error bars) by interpersonal trauma groups.

Table 3

Zero-Order Correlations Between the Total Number of Close Interpersonal Trauma Events Reported, Symptoms, and Priming

\begin{tabular}{|c|c|c|c|c|c|}
\hline & $\begin{array}{l}\text { Dissociative } \\
\text { experiences }\end{array}$ & $\begin{array}{l}\text { General } \\
\text { distress }\end{array}$ & $\begin{array}{c}\text { PTSD } \\
\text { symptom } \\
\text { severity }\end{array}$ & $\begin{array}{l}\text { Harm-to- } \\
\text { relationship } \\
\text { priming }\end{array}$ & $\begin{array}{c}\text { Relationship- } \\
\text { to-harm } \\
\text { priming }\end{array}$ \\
\hline $\begin{array}{l}\text { Total number close interpersonal } \\
\text { traumas reported }\end{array}$ & .18 & $.42^{* * *}$ & $.29^{*}$ & .07 & $.22^{*}$ \\
\hline Dissociative experiences & & $.50^{* * *}$ & $.41^{* * *}$ & .06 & .11 \\
\hline General distress & & & $.54^{* * *}$ & .15 & .12 \\
\hline PTSD symptom severity & & & & .02 & .05 \\
\hline Harm-relationship priming & & & & & $.63^{* * *}$ \\
\hline
\end{tabular}

Note. Dissociative experiences assessed by the Dissociation Experiences Scale; general distress by the Trauma Symptom Checklist; and posttraumatic stress disorder (PTSD) symptom severity by the Revised Civilian Mississippi Scale for PTSD. Missing data due to participant failure to complete questionnaires or computer problems are detailed in Table 1 .

${ }^{*} p<.05 .{ }^{* *} p<.01 .{ }^{* * *} p<.001$.

distress, dissociation, relationship-harm priming scores, and harm-relationship priming scores were entered in a simultaneous regression predicting total number of victimizations; the full model was significant, $F(4,90)=6.65$, $p<.001, R^{2}=.23$. As shown in Table 4 , only general distress and relationship-harm priming explained unique variance in total number of victimizations. The second simultaneous regression included only participants who reported some form of trauma exposure; PTSD symptom severity was added to the model. The full model was significant, $F(5,72)=4.41, p=.001, R^{2}=.23$. As in the previous regression, only general distress and relationshipharm priming explained unique variance in total number of victimizations.

\section{DISCUSSION}

Using a well-established implicit paradigm to assess schemata, women who reported exposure to multiple interpersonal traumas involving close others showed evidence of automatic relationship-harm associations that differed significantly from their singly and nonvictimized peers. This finding suggests that, when women exposed to multiple interpersonal traumas involving close others encounter information about relationships, concepts of harm are also automatically activated; however, such activation does not occur for their singly or nonexposed peers.

These findings are striking for several reasons. The current study is the first to demonstrate alterations in relationship schema using an implicit task; previous studies have relied on explicit self-reports of relationship beliefs. The findings suggest that women exposed to multiple interpersonal traumas involving close others differ from their peers in their expectations or beliefs about relationships at an automatic, nonconscious level. Specifically, the multiply exposed women performed in a way that suggests that, when they encounter information about relationships, concepts of harm are automatically activated. Such a relational schema (or automatic, nonconscious associations) is likely to have an impact on thoughts about and behaviors in relationships 
Table 4

Regression Coefficients for Model Predicting Total Number of Close Interpersonal Trauma Exposure

\begin{tabular}{llcrc}
\hline & \multicolumn{1}{c}{ Variable } & SE(B) & Beta & $t$ \\
\hline All participants & General distress & .02 & .45 & $4.20^{* * *}$ \\
& Dissociative experiences & .03 & -.07 & -.63 \\
& Harm-relationship priming & .002 & -.16 & -1.36 \\
& Relationship-harm priming & .001 & .26 & $2.20^{*}$ \\
Subset of participants & General distress & .02 & .42 & $3.16^{* *}$ \\
reporting any trauma & Dissociative experiences & .03 & -.07 & -.60 \\
& PTSD symptom severity & .48 & .09 & .72 \\
& Harm-relationship priming & .002 & -.18 & -1.32 \\
& Relationship-harm priming & .002 & .28 & $2.01^{*}$ \\
\hline
\end{tabular}

${ }^{*} p<.05 .{ }^{* *} p<.01 .{ }^{* * *} p<.001$.

(e.g., Bargh et al., 1995; Lindgren et al., 2007); therefore, these findings are important to our growing understanding of revictimization.

We also found that relationship-harm (and not harmrelationship) associations predicted unique variance in the total number of close interpersonal traumas reported by participants, even after controlling for trauma-related distress, including PTSD symptom severity and dissociative experiences. Thus, the link between the number of close interpersonal trauma events reported and automatic relationship-harm priming does not appear to operate through symptoms. These findings justify the further examination of automatic relationship-harm associations and should be considered in future studies of revictimization.

Although there was clear evidence of relationship-harm priming in the multiply victimized group that differed from the other two groups, evidence for harm-relationship priming was ambiguous. There were no differences between the groups on harm-relationship priming scores; however, the multiply victimized group showed a trend toward harmrelationship priming when their scores were compared to zero. Unidirectional relationship-harm priming is consistent with previous research on aggression in which powersex and not sex-power links have been observed among aggressive or potentially aggressive men (e.g., Bargh et al., 1995). However, the findings were not entirely consistent with previous aggression studies because multiply victimized women showed a trend toward harm-relationship priming. While this trend could suggest that priming is bidirectional in the multiply victimized group, any post hoc inferences about the trend should be drawn cautiously.

\section{Relationship-Harm Priming: Consequence of or Risk Factor for Multiple Close, Interpersonal Trauma Exposures?}

Like other cross-sectional studies of priming (e.g., Bargh et al., 1995), we cannot determine whether automatic relationship-harm associations are a consequence of or a risk factor for multiple close interpersonal trauma exposures. Logically, however, it makes sense that, as the number of close interpersonal trauma exposures increase, women learn to expect that close relationships involve harm. The automatic relationship-harm associations observed in the multiply victimized group therefore may be a consequence of learning that takes place in the context of such repeated exposure.

Once learned, these automatic relationship-harm associations may increase the likelihood that women expect harm in later relationships and, therefore, behave differently from their peers. For example, women who associate relationships with harm may be more likely to stay in a relationship that becomes violent and/or feel disempowered to leave such a relationship. Conceptualizing the relationshipharm associations as a risk factor for additional victimizations is consistent with the interpersonal schema hypothesis of revictimization (Cloitre, 1998; Cloitre et al., 2002).

\section{Limitations}

Implicit tasks offer insight into automatic beliefs and associations that operate outside of the individual's conscious awareness (see Greenwald \& Banji, 1995); however, the external validity of such tasks should be considered in interpreting findings. Although these data are supportive of the interpersonal schema hypothesis, we do not know the extent to which such automatic associations translate into behaviors in interpersonal relationships.

We did not have adequate representation across different types of traumas (e.g., sexual assault vs. physical assault, close vs. not-close victim-perpetrator relationship) to explore associations between particular types of trauma exposure and priming. An important next step in this line of research, particularly as efforts seek to clarify whether alterations in automatic associations are a correlate or consequence of trauma exposure, will be to use recruiting procedures that allow for comparisons across a range of categories of trauma exposure.

\section{Conclusions}

The current study provides an implicit test of automatic relationship-harm associations in college women. This 
research is an important addition to previous methods that have relied on participants' explicit awareness of relationship schemas (e.g., survey methods that ask participants to report on beliefs about relationships) by capturing automatic associations outside of conscious awareness. In light of the urgent need to improve intervention efforts targeting revictimization risk, the current study highlights the importance of considering relationship schemata as either a consequence of or risk factor for revictimization.

Initial submission: April 2, 2007

Initial acceptance: April 25, 2008

Final acceptance: June 16, 2008

\section{NOTE}

1. A complete list of stimuli is available from the first author.

\section{REFERENCES}

Arata, C. M. (2002). Child sexual abuse and sexual revictimization. Clinical Psychology: Science and Practice, 9, 135-164.

Bargh, J. A., Raymond, P., Pryor, J. B., \& Strack, F. (1995). Attractiveness of the underling: An automatic power $\rightarrow$ sex association and its consequences for sexual harassment and aggression. Journal of Personality and Social Psychology, 68, 768-781.

Bernstein, E. M., \& Putnam, F. W. (1986). Development, reliability, and validity of a dissociation scale. Journal of Nervous and Mental Disorders, 174, 727-735.

Briere, J., \& Runtz, M. (1989). The Trauma Symptom Checklist (TSC-33): Early data on a new scale. Journal of Interpersonal Violence, 4, 151-163.

Bushman, B. J. (1998). Priming effects of media violence on the accessibility of aggressive constructs in memory. Personality and Social Psychology Bulletin, 24, 537-545.

Classen, C. C., Palesh, O. G., \& Aggarwal, R. (2005). Sexual revictimization: A review of the empirical literature. Trauma, Violence, \& Abuse, 6, 103-129.

Cloitre, M. (1998). Sexual revictimization: Risk factors and prevention. In V. M. Follette, J. I. Ruzek, \& F. R. Abueg (Eds.), Cognitive-behavioral therapies for trauma (pp. 278-304). New York: Guilford.

Cloitre, M., Cohen, L. R., \& Scarvalone, P. (2002). Understanding revictimization among childhood sexual abuse survivors: An interpersonal schema approach. Journal of Cognitive Psychotherapy: An International Quarterly, 16, 91-111.

Cloitre, M., Tardiff, K., Marzuk, P. M., Leon, A. C., \& Potera, L. (1996). Childhood abuse and subsequent sexual assault among female inpatients. Journal of Traumatic Stress, 9, 473-482.

DePrince, A. P. (2005). Social cognition and revictimization risk. Journal of Trauma and Dissociation, 6, 125-141.

DePrince, A. P., \& Freyd, J. J. (2004). Forgetting trauma stimuli. Psychological Science, 15, 488-492.

Elliott, D. M., \& Briere, J. (1992). Sexual abuse trauma among professional women: Validating the Trauma Symptom Checklist-40 (TSC-40). Child Abuse and Neglect, 16, 391398.

Freyd, J. J., DePrince, A. P., \& Gleaves, D. (2007). The state of betrayal trauma theory: Conceptual issues, critiques, future directions. Memory, 15, 295-311.
Gidycz, C. A., Hanson, K., \& Layman, M. J. (1995). A prospective analysis of the relationships among sexual assault experiences: An extension of previous findings. Psychology of Women Quarterly, 19, 5-29.

Goldberg, L. R., \& Freyd, J. J. (2006). Self-reports of potentially traumatic experiences in an adult community sample: Gender differences and test-retest stabilities of the items in a Brief Betrayal-Trauma Survey. Journal of Trauma \& Dissociation, 7(3), 39-63.

Greenwald, A. G., \& Banaji, M. R. (1995). Implicit social cognition: Attitudes, self-esteem, and stereotypes. Psychological Review, 102, 4-27.

Holland, R. A., Hendriks, M., \& Aarts, H. (2005). Smells like clean spirit: Nonconscious effects of scent on cognition and behavior. Psychological Science, 16, 689-693.

Jurgens, H. J. (2005). Interpersonal schemas and functioning in women abused in childhood: The role of revictimization. Graduate Faculty Psychology Bulletin, 3, 11-41.

Kamphuis, J. H., deRuiter, C., Janssen, B., \& Spierling, M. (2005). Preliminary evidence of an automatic link between sex and power among men who molest children. Journal of Interpersonal Violence, 20, 1351-1365.

Lindgren, K. P., Shoda, Y., \& George, W. H. (2007). Sexual or friendly? Associations about women, men, and self. Psychology of Women Quarterly, 31, 190-201.

Loftus, G. R. (1996). Psychology will be a much better science when we change the way we analyze data. Current Directions in Psychological Science, 1996, 161-171.

McKenna, F. P., \& Sharma, D. (1995). Intrusive cognitions: An investigation of the emotional Stroop task. Journal of Experimental Psychology: Learning, Memory, \& Cognition, 21, 1595-1607.

McNally, R. J., Metzger, L. J., Lasko, N. B., Clancy, S. A., \& Pitman, R. K. (1998). Directed forgetting of trauma cues in adult survivors of childhood sexual abuse with and without posttraumatic stress disorder. Journal of Abnormal Psychology, 107, 596-601.

Meyer, D. E., Schvaneveldt, R. W., \& Ruddy, M. G. (1975). Loci of contextual effects on visual word recognition. In P. M. A. Rabbit \& S. Dornic (Eds.), Attention and performance: $V$ (pp. 98-118). London: Academic Press.

Mussweiler, T., \& Forster, J. (2000). The sex $\rightarrow$ aggression link: A perception-behavior dissociation. Journal of Personality and Social Psychology, 79, 507-520.

Noll, J. G., Horowitz, L. A., Bonanno, G. A., Trickett, P. K., \& Putnam, F. W. (2003). Revictimization and self-harm in females who experienced childhood sexual abuse. Journal of Interpersonal Violence, 18, 1452-1471.

Norris, F. H., \& Perilla, J. L. (1996). The Revised Civilian Mississippi Scale for PTSD: Reliability, validity, and crosslanguage stability. Journal of Traumatic Stress, 9, 285298.

Vitevitch, M. S. (2007). The spread of the phonological neighborhood influences spoken work recognition. Memory \& Cognition, 35, 166-175.

Williams, J. M. G., Mathews, A., \& MacLeod, C. (1996). The emotional Stroop task and psychopathology. Psychological Bulletin, 120, 3-24.

Zurbriggen, E. L. (2000). Social motives and cognitive powersex associations: Predictors of aggressive sexual behavior. Journal of Personality and Social Psychology, 78, 559581. 